\title{
Extreme Space Weather Events during the First Cycles of Epochs with Decreased Solar Activity
}

\author{
V. N. Ishkov* \\ Pushkov Institute of Terrestrial Magnetism, Ionosphere, and Radio Wave Propagation, \\ Russian Academy of Sciences, Moscow, Troitsk, Russia \\ *e-mail: ishkov@izmiran.ru
}

Received March 8, 2021; revised April 21, 2021; accepted May 27, 2021

\begin{abstract}
The problem of the distribution of extreme and very strong magnetic storms with intensities (G5, G4) in the first cycles (12 and 24) of epochs of lowered solar activity was considered based on homogeneous series of the geomagnetic index $A a$ with allowance for the modern scale of the intensity of disturbances in the near-Earth space and the scenario of solar cyclicity. The significant decrease in the number of such events and active solar phenomena in the last cycle may indicate that the sunspot and flare activity in solar cycle 12 was significantly higher than that in cycle 24 , but it was significantly lower than in solar cycles of the epoch of increased solar activity.
\end{abstract}

DOI: $10.1134 / \mathrm{S} 0016793221060074$

\section{INTRODUCTION}

\subsection{Reliable Time Series of Solar-Activity Observations}

One of the most remarkable features of the Sun are the almost periodic changes in various manifestations of solar activity (SA), the entire set of observed, nonstationary phenomena in the Sun's atmosphere. These phenomena cause changes in its radiation in different ranges of electromagnetic waves and in particle fluxes of different energies. The SA level is characterized by several observational indices. The index longest series is the relative sunspot number (W), which was first introduced by R. Wolf in 1849 when a service was organized in Europe: daily sunspot observations with one method at several observatories. Thus, scientific observations of the Sun began. In addition, Wolf restored the monthly mean values of this index from 1749 and the annual mean values from 1700 based on incomplete observational data. The SA cycle beginning in March 1755 was conventionally adopted as the first cycle, thereby forming a "numbered" series of relative sunspots. It should be kept in mind that, until 1849 , this series was reconstructed from rather fragmentary data from various European observers. Modern researchers, using even more fragmentary data from individual telescopic observations, were able to extend this series qualitatively to 1611 . The reliability of all of the reconstructed data is incomparably lower than reliable series: the spectral characteristics of the reconstructed part (100 years) are completely different from reliable series, and it contains features and characteristics of individual cycles that have not been observed in a reliable series over 180 years (Ishkov and Shibaev, 2006).

Thus, 15 reliable SA cycles are available for scientific research. Shibaev and Ishkov (2012) gave arguments for the possible inclusion (for a limited range of problems) of cycles 8 and 9 (as conditionally reliable), which differ the least in their main characteristics from reliable solar cycles (SCs). Thus, we have expanded the statistics to 17 SCs (1834-2021).

\subsection{Solar-Activity Cycles}

The SA cycles are manifested by a regular, almost periodic change in the number of sunspots and other active formations observed on the visible disk of the Sun. The complete, physical SA cycle is associated with the dynamics of the solar magnetic field, has an average duration of 22 years, and consists of two 11 -year cycles. The first cycle has an even number and usually has a lower amplitude.

The mean period of significant reliable cyclical changes is 10.81 years, although it has a tendency to decrease. Thus, in seven solar cycles of the 20th century (cycles 15-19, 21, 22), the average cycle duration was 10.44 years. At present, the statistics of sunspot observations makes it possible to determine the scenario of solar cyclicity, its properties and characteristics, and developmental laws for reliable (10-24 cycles) and conditionally reliable solar cycles (8 and 9) on a time scale of 180 years (Fig. 1). A reliable series of relative sunspot numbers shows surprising consistency in the main manifestations of the development of individual 


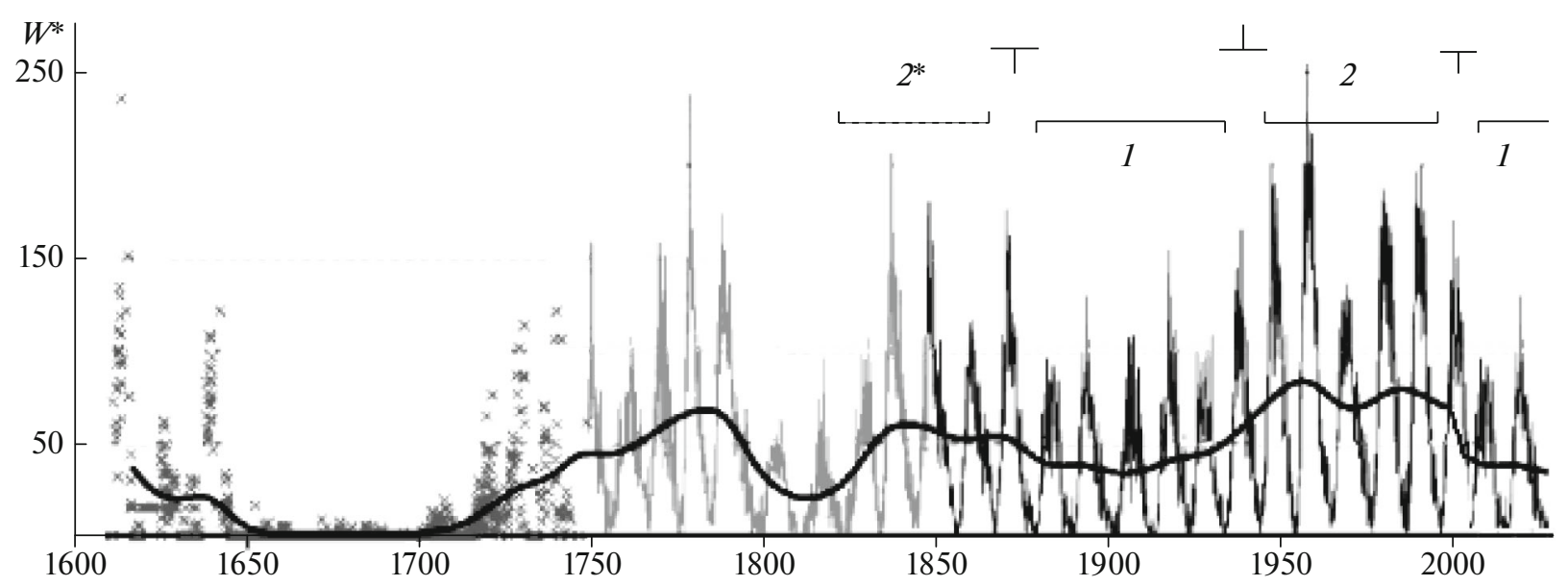

Fig. 1. Complete series of observations of the relative sunspot numbers. A reliable series from 1849 (black) with the boundaries of structural epochs of lowered (1) and increased (2) SA with transitional cycles between them. Probable period of increased solar activity $\left(2^{*}\right)$, including SCs 10-6. Probable course of development of SC 25 (last on the right). Converted picture from Wikipedia http://en.wikipedia.org/wiki/Sunspot.

11- and 22-year cycles. The structure of the cyclicity of a reliable series based on this statistic involves a periodic change of epochs with increased (SC 6, 7, 8-10 and 1822) and lowered SA 10 (SC 12-16 and 24-281). Transitional solar cycles $(11,23$, and 17) are observed between these epochs: periods during which the character of sunspot-forming activity, i.e., the modes of the generation of magnetic fields in the sunspot-forming zone of the Sun, changes (Ishkov, 2013; Ishkov, 2018).

The first epoch of lowered solar activity lasted from 1878 to 1933 (five solar cycles). At that time, however, observational data on the Sun were limited to the Wolf numbers, the number and areas of sunspot groups, and data on variations in the geomagnetic field: the magnitude of the disturbance of the geomagnetic field (the $A a$ index) at two midlatitude geomagnetic observatories spaced across the hemispheres that continuously record the state of the Earth's magnetic field and sudden short-term increases in the geomagnetic field (SC, sudden commencement), which (in the modern sense) reflect the arrival of interplanetary shock waves into near-Earth space (NES). In addition, according to the records of geomagnetic-field variations, one can distinguish the so-called "crochet" (from the French crochet, "hook"), which appears at the moment of irradiation of the Earth's ionosphere by electromagnetic radiation from the Sun at the maximum of a sufficiently powerful solar flare. However, even from these observations, it is possible to estimate the course of development and, to some approximation, the characteristics of the SC flare activity and its geoeffectiveness in the epoch of lowered SA. Observations of the Sun and its influence on the NES became regular and more complete only from the beginning of the

\footnotetext{
${ }^{1}$ Italic font denotes the cycles not included into the family of reliable or currently not reliable.
}

space age (SC 19, the early 1960s). The bulk of our knowledge about active phenomena on the Sun and their influence on the NES relates to the epoch of increased SA (1944-1996) and the transition period SC23 between epochs, when, after the weakening (by more than two times) of the total magnetic field of the Sun, almost all of the magnetic characteristics of the active phenomena on the Sun decreased during the SC 24 period, and, as a consequence, the magnetic fields in the surrounding interplanetary space, and, accordingly, in the NES decreased (Ishkov, 2018). Hence, it became possible only in SC 24 (the first SC of the second epoch of lowered SA and in the current SC 25) to study the main characteristics of the geoeffective activity of the first epoch of lowered SA, for both background and average values. The latter, according to the scenarios of reliable SCs, should be of average value, since the Gnevyshev-Ol' rule was strictly observed within the epochs on a reliable series. Violations of this rule can only be expected during transition periods, as happened in SCs 22-23. Observations show that these two SCs were quite unusual in their characteristics and to some extent violated the established pattern of SC development. As SC 23 developed, it became clear that we are observing processes associated with the restructuring of the conditions of sunspot-forming activity from an epoch of increased SA to an epoch of lowered SA (Ishkov, 2013), which directly depend on the changes in the general magnetic field of the Sun.

This work is based on the following observational data:

- a reliable series of relative sunspot numbers in 1849-2019, version V1 (http://sidc.oma.be/silso/ datafiles), with stipulation of the use of version V2;

- a reliable corrected series of areas of sunspot groups (1874-2019) (http://fenyi.solarobs.csfk.mta. hu/GPR/); 
- the modern scale of the impact of solar-activity phenomena on the NES (http://www.swpc.noaa.gov/ NOAAscale);

- a complete series of the longest observations of the geomagnetic activity (aa index) in 1868-2019 (http://isgi.unistra.fr);

- a complete series of the registration of the sudden commencement of geomagnetic storms in 18682019 (http://isgi.unistra.fr);

- the catalog of large sunspots and geomagnetic storms from the Greenwich Observatory in 1874-1954 (Sunspot and geomagnetic storm data, 1955)

The goal of this work is to compare the conditions for the realization of geoeffective solar phenomena and their extreme manifestations in the NES in the initial SC epochs of lowered SA.

\section{MODERN SCALE OF THE IMPACT OF SOLAR-ACTIVITY PHENOMENA} AND EXTREME EVENTS IN THE NES

The modern scale of the impact of solar-activity phenomena on the NES evaluates such a reaction with a five-point, numbered, level scale that increases in intensity at three positions (event types): R, S, and G (https://www.swpc.noaa.gov/noaa-scales-explanation):

$R(1-5)$. An electromagnetic shock is the impact of electromagnetic radiation during the development of a powerful solar flare, mainly on the ionosphere (sudden ionospheric disturbances), which leads to disruption of radio communications in a time interval of up to several hours. The recording of such events became possible only with the advent and development of applied radio communication, i.e., approximately from the first third of the 20th century. However, in terms of ionospheric effects (solar flare effect, sfe), this became possible in a first approximation from the beginning of service observations of geomagnetic-field variations.

$S(1-5)$. Solar proton events (SPE) are the arrival of high-energy ( $E \geq 10 \mathrm{MeV})$, charged, solar particles at the NES, which affect the radiation environment in the vicinity of the Earth, create new and strengthen existing radiation belts, cause an increase in the electron concentration over the polar caps, and disrupt radio communication at the polar tracks. Continuous SPE observations became possible only with the beginning of the era of space exploration, when it became possible to record charged particles in the entire energy range. It should be noted that S5-class events with a proton flux of $\geq 10^{5} \mathrm{~cm}^{-2} \mathrm{~s}^{-1} \mathrm{sr}^{-1}$ have not yet been observed.

$G$ (1-5). Disturbances of the geomagnetic field (magnetic storms) are a consequence of the arrival at the NES of solar plasma streams of increased density, particle velocity and temperature, with an enhanced magnetic field. The intensity of magnetic storms in this position is estimated with the planetary $3-\mathrm{h} K p$ index, which is calculated from the $K p$ indices of 12 ground-based, mid-latitude, magnetic observatories distributed over the globe.

The extreme events in this article include events that fall into positions (classes) G4 and G5.

\subsection{Extreme Solar Events and Their Manifestation in the Near Earth Space}

The definition of extreme events (Ishkov, 2015) directly depends on those disturbances (extreme deviations from background values) in the NES that produce solar flare events: flares, ejections of solar filaments, which are the same flares but outside active regions in weak magnetic fields. By themselves, solaractivity phenomena, even of maximum intensity and sizes, are usually of interest only to a narrow circle of solar physicists if they do not cause strong magnetic storms or powerful solar proton events. Thus, it would be natural to define extreme solar events as large powerful flare phenomena, the consequence of which in the NES is the occurrence of maximum perturbations in all three positions, i.e., R4-5, S4-5, and G4-5. However, phenomena of the $\mathrm{S}$ and $\mathrm{G}$ positions substantially depend on the localization of the solar flare event on the visible solar disk. For example, disturbances of the geomagnetic field $(\mathrm{G})$ from a powerful flare event near the western limb of the Sun would be minimal, since a larger fraction of the energy of the coronal mass ejection (CME) from this flare would escape the Earth (flare event of November 4, 2003). The most powerful flare on the eastern limb will be weakly manifested in both $\mathrm{S}$ and $\mathrm{G}$, since both solar protons and the CME would be directed away from the Earth (events of January 6, 1991, June 3, 1989). Before the space age, the most powerful solar flare events could only be estimated by the effects they caused in the NES. Observers often noted that such effects were associated with flares, the radiation from which was observed in a continuous spectrum ("white light"), i.e., during the observation of sunspot groups on the photosphere. A solar extreme flare event was observed for the first time by R. Carrington on September 1, 1859, as a flare in "white light" (Carrington, 1859). After $17 \mathrm{~h} 40 \mathrm{~m}$ a severe magnetic storm was then noted in America and Europe, after which serious violations of wire telegraph communication, and the auroras were observed in Rome, Havana, and Hawaii. Similar flares were noted in late January-early March 1942 in SC 17, in November 1949 in SC 18, in November 1960 and possibly in July 1961 in SC 19, in August 1972 in SC 20, in June, July, and December 1982, and end of April 1984 in SC 21.

\subsection{Definition of Magnetic Storms and Geomagnetic-Activity Indices}

The most spectacular manifestations of the impact of solar flare events on the Earth's surface are sporadic 
magnetic storms; with the development of civilization and the emergence of high-tech systems, both terrestrial and space systems, their impact becomes more and more tangible. During severe and extreme magnetic storms, disruptions (in some cases catastrophic) are noted in technological systems, extended power systems, and pipelines; radio communications and GPS locations are disrupted, and auroras are observed in the equatorial regions. The recording of magnetic disturbances became possible after the appearance of instruments for permanent observation of the Earth's magnetic field and an expanded network of geomagnetic observatories, since 1867 . The quantitative measures of geomagnetic activity at midlatitudes are indices. Among them the planetary $A a$ index is first in terms of the duration of a reliable series of observations. It is determined based on data from two magnetic observatories in different hemispheres of the Earth. Then, since 1932, the daily planetary index $A p$ became the main index. It is determined via the averaging of eight 3-h values of the ap index of 12 terrestrial, midlatitude (between $63^{\circ} \mathrm{N}$ and $48^{\circ} \mathrm{S}$ geomagnetic latitudes), magnetic observatories all over the globe. From 1947, the Dst index, which gives the change in the magnetic field due to the appearance of a ring current in the magnetosphere is introduced. The Dst index is calculated from the values of the $H$ component of the field of four observatories that are evenly distributed by longitude and not affected by the auroral and equatorial electric jets.

The G-class of magnetic disturbances is based on the planetary 3-h $K p$ index, which is calculated via simple averaging of the values of 12 magnetic observatories, has a 27-point scale of disturbance increase, and is expressed by numbers from 0 to 9 with signs -, $0,+$ from $0 \mathrm{o}$ to $9 \mathrm{o}$. Position $0 \mathrm{o}$ indicates an extremely quiet period, while $9 \mathrm{o}$ is the mostly intense storm. The intensity of the magnetic disturbance during the period of its implementation is estimated by the highest value of the $K p$ index, which leads the entire $\mathrm{G}$ scale to an estimate of the maximum intensity of the phenomenon, not to an estimate of its power. Within the $\mathrm{G}$ class, the magnetic-storm intensity is estimated as follows: G5, if at least one 3-h interval has $K p=9$, extreme magnetic storm; G4, severe, $K p=8$; G3, strong, $K p=7 ; \mathrm{G} 2$, moderate, $K p=6$; G1, minor, $K p=5$.

However, one should keep in mind that the $K p$ index has a semilogarithmic scale and that it is more convenient in real work to use the $A p$ index, the average daily planetary amplitude in nT of the most disturbed magnetic element $D$ or $H$, which is obtained via the averaging of eight individual $a p$ values (corresponding to $K p$ ) in 3-h intervals per day. Thus, the $A a$ index is a simpler analog of the $A p$ index for two midlatitude magnetic observatories. Accordingly, to estimate the intensity of magnetic disturbance in the recording system of the $A a$ index, we will use reliable series of the $K p a$ index, an analog of $K p$, the International Geomagnetic Index Service (http://isgi.unistra.fr), which measures the amplitude of global geomagnetic activity in a 3-h interval, reduced to geomagnetic latitudes of $\pm 50^{\circ}$.

The correlation between them over the general time interval is on average 0.9 , although it is slightly weaker in even SCs and stronger in the phases of the minimum and in the daytime (Mayaud, 1972; Coffey and Erwin, 2001). This result makes it possible to compare significant magnetic disturbances in the $K p a$-index system in different SCs of the entire reliable series of solar observations.

Since most works on space weather use the NOAA scale (https://www.swpc.noaa.gov/noaa-scales-explanation) to assess the impact of disturbances on the NES, we will remain within its framework in this work. However, in our opinion, there is a more objective criterion for the assessment of the power of magnetic storms (Gosling et al., 1991). The corresponding indices should be recorded at least in three 3-h intervals per day: ( $K p_{\max } \geq 8$ and $K p \geq 6$ ) for severe magnetic storms indices; $\left(7-\leq K p_{\max } \leq 7+\right.$ and $K p \geq 6$ ) for strong storms; moderate storms are all events with $K p$ of at least 6-; and 5- $\leq K p_{\max } \leq 5+$ for minor storms.

In this work, the term "magnetic storm" is understood as a magnetic disturbance lasting at least $12 \mathrm{~h}$ and the average index $A p(A a)$ of the disturbance reaches 27 (in Western countries, the threshold value is $A p=30$ ). In addition, a magnetic storm is not interrupted by a single value, $K p=3(-/ 0 /+)$; for example, the series- $(4+5+305-4-5-3-5-40)$ describes one magnetic storm and one event. Magnetic disturbances lasting less than $12 \mathrm{~h}$ (three 3 -h intervals) in this work refer to midlatitude magnetic substorms.

\section{EXTREME MAGNETIC STORM DURING THE FIRST CYCLES OF EPOCHS OF LOWERED SOLAR ACTIVITY}

It has become clear that both powerful flare events and flare events of middle classes $(<\mathrm{M} 5)$ with complex internal development can be sources of extreme magnetic storms. They occur in flare-activity regions geoeffectively located on the visible disk of the Sun. Highspeed CMEs of the "halo" type, which occupy an angle of $360^{\circ}$ in the field of view, are always a component of these flare events. The "partial halo" CME type $\left(90^{\circ}-300^{\circ}\right)$ are components more rarely. A good example of a source of an extreme magnetic storm is the event of November 18, 2003, when two X-ray bursts of the M3.2 and M3.9 classes occurred within the framework of one optical flare $(2 \mathrm{~N})$ with a filament ejection that occurred between them (Srivastava et al., 2009). All three "subevents" were realized in a time interval of $\sim 2 \mathrm{~h}$ and were accompanied by three CMEs. The interaction of these CMEs in the interplanetary space caused the highest intensity (G5: $K p=9$, $D s t=-472 \mathrm{nT})$, but not the most powerful, magnetic storm in the NES in SC 23. Therefore, in the determi- 
nation of the source of extreme disturbance, it is necessary, whenever possible, to take into account the entire dynamics of the development of this event, both on the Sun and in the interplanetary space.

As previously noted, the SC 22 decline branch and the entire SC 23 constituted a transition period between epochs of increased and lowered SA, when the background values of the total solar magnetic field significantly decreased (Svalgaard, 2011). This led to a change in the sunspot generation in the convective zone. This process led to a decrease in the magnetic field in all structures of active phenomena on the Sun from sunspots (which was reflected in the flare activity) to coronal holes. This resulted in recurrent events, and, accordingly, for all magnetic and physical characteristics of the solar wind, a significant reduction in the level of the geoeffectiveness of their impact on the NES. The most representative consequence of this process was the observed decrease in the magnetic field and the corresponding increase in brightness in the sunspot umbra (Livingston et al., 2012).

It is natural to expect that a similar process of a gradual decrease in the background values of the total magnetic field of the Sun also occurred before the onset of SC 12, in the same transition period from an epoch of increased SA to an epoch of lowered SA from mid-SC 10 to the end of SC 11. Considering highintensity magnetic disturbances and linking them to the situation on the Sun, one can, in some approximation, compare the geoeffectiveness of solar-activity phenomena and the state of the interplanetary medium and can draw preliminary conclusions about the level of the background general magnetic field of the Sun in the initial cycles of epochs with low SA.

The initial cycle of the first epoch of low SA (SC 12) began in December 1878 with an initial value of $W_{\text {min }}^{*}=2.2$ (3.7 in the $\mathrm{V} 2$ version), with a maximum of $W_{\max }^{*}=74.6$ (124.4) in December 1883 and ended in February 1890 at a value of $W_{\min }^{*}=5.0(8.3)$. The rise branch took 5.0 years, and the decline branch took 6.3 years, which ensured a full cycle-development time of $11.3 \mathrm{yr}$. The first large sunspot group $\left(S p_{\max }=\right.$ 1155 millionth of solar hemisphere, msh) appeared in the northern hemisphere at the end of May 1880, 1.5 years after the beginning of the cycle; the first is very large in April $1882\left(S p_{\max }=2258 \mathrm{msh}\right)$. In total, 11 very large sunspot groups appeared during the cycle, eight on the rise branches and three on the decline branches.

The first cycle of the second epoch of lowered SA (SC 24) began in January 2009 with an initial value of $W_{\min }^{*}=1.7(2.2$ in version $\mathrm{V} 2)$, with a maximum of $W_{\max }^{*}=81.9$ (116.4) in April 2014 and ended in December 2019 at an even lower value $W_{\min }^{*}=1.3(1.8)$. The rise branch lasted 5.3 years, the decline branch was 5.7 years, and the total duration of SC 24 was 11.0 years.
The first large sunspot group ( $S p=550 \mathrm{msh}$ ) appeared in the northern hemisphere in October 2010, 1.8 years after the beginning of the cycle. However, a very large (1560 msh) sunspot group, which remained the same size during two solar revolutions (1580 $\mathrm{msh})$, appeared only in January 2014, like the only giant ( $S p=2740 \mathrm{msh}$ ) in October 2014, and was entirely in the southern hemisphere. Note that not a single giant sunspot group $(S p \geq 2500 \mathrm{msh})$ was noted in SC 12, although there were four very large sunspots with larger areas $(S p \sim$ $2000 \mathrm{msh}$ ). It should be noted that the course of the development of the considered solar cycles, is largely similar, as can be seen from Fig. 2, and their durations are almost the same. Both cycles (SCs 12 and 24) have two significant peaks, and the second peaks were the cycle maxima. In both SCs, up to about half of the rise branch, sunspot groups of the northern hemisphere prevailed, and the main maxima were provided by the sunspot-forming activity of the southern hemisphere (http://users.telenet.be/j.janssens/SC24web/SC24.html\#Hemarea). However, the entire decline branch in SC 12 was dominated by sunspot groups of the southern hemisphere, while activity in the northern hemisphere again prevailed in the minimum phase in SC 24.

A complete comparison of all of the characteristics and the development of the first SC and of both epochs of lowered SA is a task for future studies, as observations on the further development of the second such epoch accumulate, but let us now limit ourselves to a comparison of the occurrence of significant magnetic disturbances and, especially, extreme magnetic disturbances in them, especially the extreme magnetic storms (G5, G4) given in Table 1. The time of the beginning, maximum, and end of the magnetic disturbance is given at the beginning of the 3-h interval of the Kpa index (http://isgi.unistra.fr). Sunspot groups in which a flare event, a source of magnetic disturbance, most likely took place during SC 12 (Sunspot and geomagnetic storm data, 1955) are given for each event.

According to this catalog, two of sunspot groups with flare events are sources of extreme magnetic storms in SC 12; they had very large areas exceeding 2000 msh (Fig. 3). Flare events that caused two magnetic storms, one in November and one in April, occurred in sunspot groups Gr885 and Gr729. They were considered, since the intensity of the second April group belonged to the G3 class. The sources of the other three extreme magnetic storms, according to the version of this catalog, were flare events that occurred in sunspot groups with areas less than $500 \mathrm{mph}$. We discussed earlier an example of an extreme magnetic storm of November 2003, the strongest in intensity in SC 23, both in the $A p$ index and Dst (-472 nT) values. This magnetic storm was caused by a complex, threestage flare event of average magnitude that occurred in sunspot group AR10501 with a sunspot area of $S p=$ $410 \mathrm{msh}$ and with a complex evolutionary and magnetic structure. It is likely that we are dealing with a 


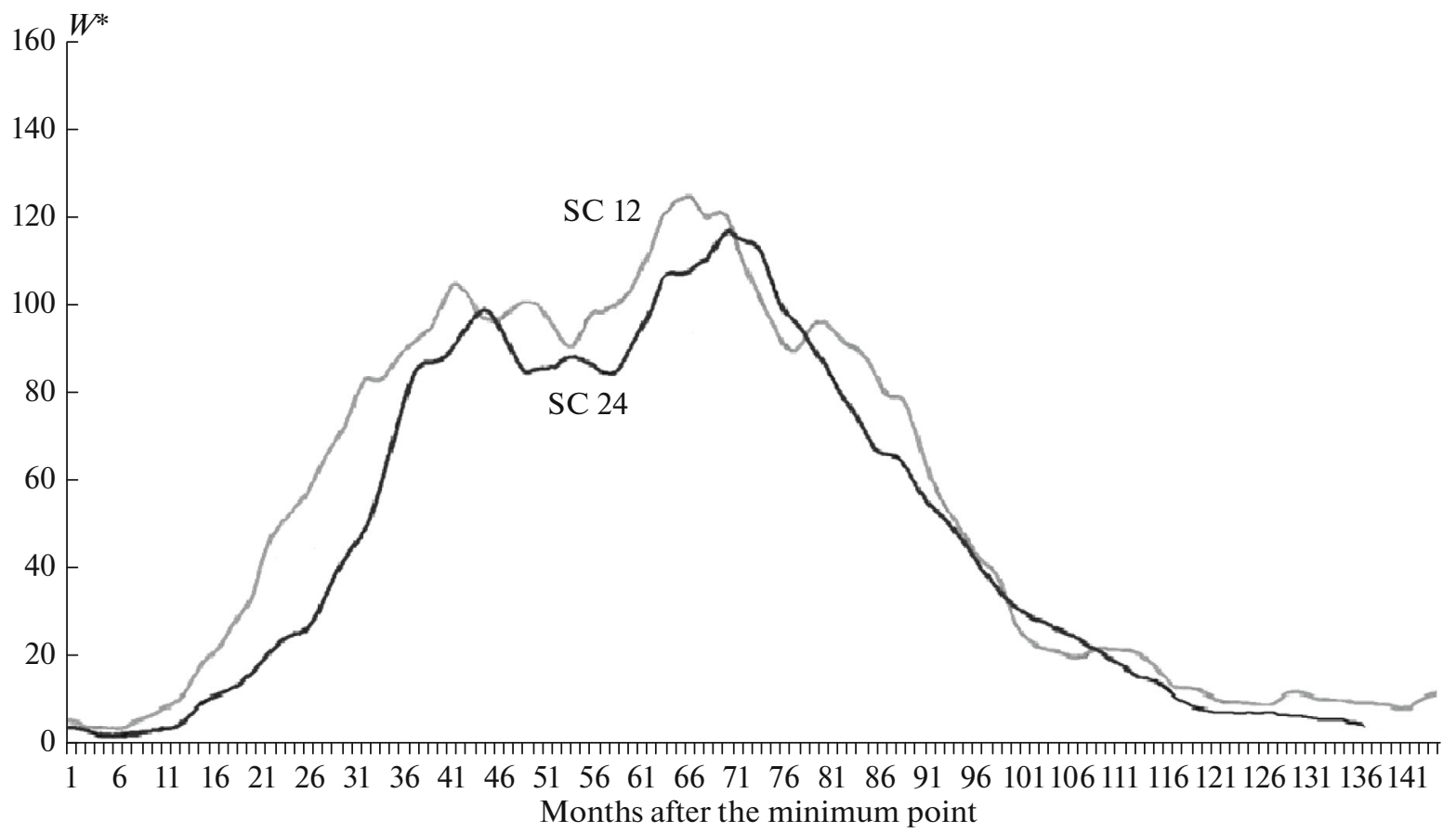

Fig. 2. The time history of SC 12 and 24 . The $W^{*}$ values are given in the new version V2 (http://sidc.oma.be/silso).

similar development of events in the case of SC 12, since that happened during the only the extreme magnetic storm in SC 24 in June 2015. The active region AR12371 (Fig. 4) passed the central meridian of the visible solar disk on June 22, 2015, and had a complex structure in the middle part of the sunspot group and a maximum area of $S p=1180 \mathrm{msh}$. A detailed study of this active region, the geoeffective flare event, and the NES response to it (Vemareddy, 2017; Piersanti et al., 2017; Joshi et al., 2018) showed that there was also a complex interaction of CMEs in this case during propagation through interplanetary space from two solar flare events: a solar filament ejection on June 19 at 0500 UT from the southeastern quadrant of the visible solar disk and a flare event on June 21 at 0102 UT, importance $1 \mathrm{~N}$, with two successive X-ray bursts of the middle classes M2.0 and M2.6, which occurred in the 1 -h interval in the middle part of the active region (Fig. 4). The disturbance from the first event entered the NES in the form of a sudden impulse (SI) on June

Table 1. Main characteristics of extreme magnetic storms of the first solar-cycle epochs of low solar activity

\begin{tabular}{|c|c|c|c|c|c|c|c|c|}
\hline № & $\begin{array}{c}\text { To } \\
\text { Year month day/hour }\end{array}$ & $\begin{array}{c}\text { Tmax } \\
\text { day/hour }\end{array}$ & $\begin{array}{c}\mathrm{Te} \\
\text { day/hour }\end{array}$ & $\begin{array}{c}\Delta \mathrm{T} \\
\text { hours }\end{array}$ & $\begin{array}{l}\text { Kpa } \\
\mathrm{nT}\end{array}$ & $\begin{array}{l}\text { Class } \\
\text { NOAA }\end{array}$ & $\begin{array}{c}\mathrm{Sc} \\
\text { day/hour/min }\end{array}$ & $\begin{array}{c}\text { AO } \\
\text { Gr, NOAA }\end{array}$ \\
\hline \multicolumn{9}{|c|}{$\underline{\mathrm{SC} 12}(1879 \mathrm{I}-1883 \mathrm{XII}-1890 \mathrm{II}) ; \mathrm{G} 5-3 \mathrm{G} 4-3 \mathrm{G} 3-11 \mathrm{G} 2-43 \mathrm{G} 1-78 ;$ Sc 212} \\
\hline 1 & $18800812 / 09$ & $12 / 12$ & $14 / 06$ & 45 & 334 & G4 & $12 / 1136$ & Gr343 \\
\hline 2 & $18810131 / 06$ & $31 / 18$ & $01 / 03$ & 21 & 337 & G4 & $31 / 0848$ & Gr412 \\
\hline 3 & $18820416 / 12$ & $17 / 12$ & $17 / 18$ & 30 & 536 & G5 & $16 / 2312$ & Gr729 \\
\hline 4 & 18821116/09 & $17 / 09,15$ & $19 / 03$ & 66 & $531 / 531$ & G5 & $16 / 0818$ & Gr885 \\
\hline & & $18 / 00-03$ & & & 464 & & $17 / 1006$ & \\
\hline 5 & $18821119 / 12$ & $20 / 09-12$ & $21 / 00$ & 36 & 536 & G5 & & Gr885 \\
\hline \multicolumn{9}{|c|}{ 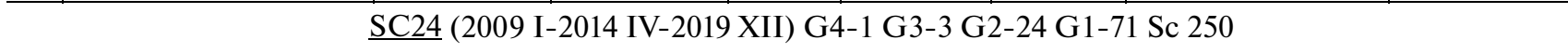 } \\
\hline 1 & $20150622 / 06$ & $22 / 18$ & $24 / 06$ & 48 & 363 & & $\begin{array}{c}22 / 0544, \\
22 / 1833\end{array}$ & AR12371 \\
\hline
\end{tabular}

To is the year, month, day, and hour of the onset; Tmax is the day and hour of the maximum values; Te is the end; $\Delta \mathrm{T}$ is the duration; the $K p a$ index is in nT; the class is given according to the NOAA scale; Sc is the day and time of arrival of a sudden pulse (sudden commencement); $\mathrm{AO}$ is the number of the sunspot group in which the flare event-source of magnetic disturbance occurred (for SC 12 is the most probable one): $\mathrm{Gr}$ is by Greenwich observatory number, AR is the NOAA number. The time is Universal Time (UT). 


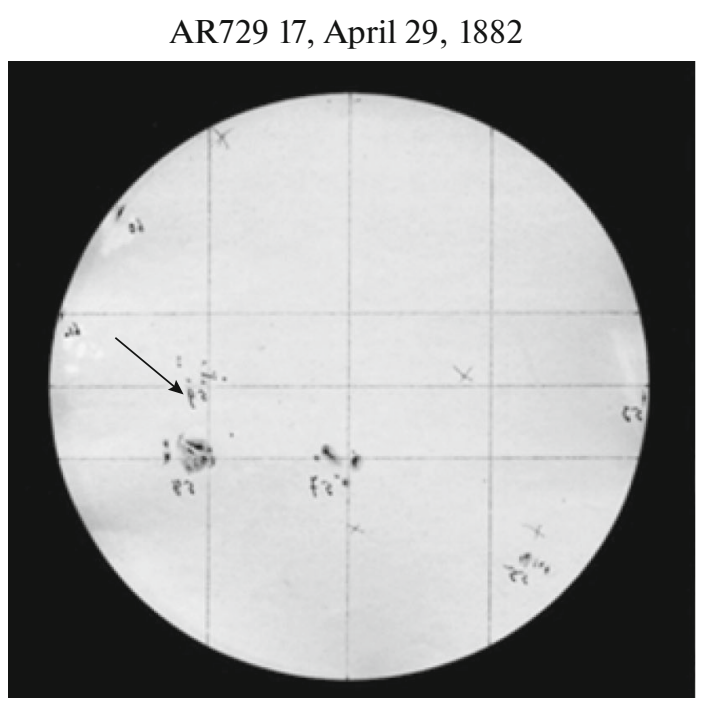

AR885 (N19L121)19, November, 1882

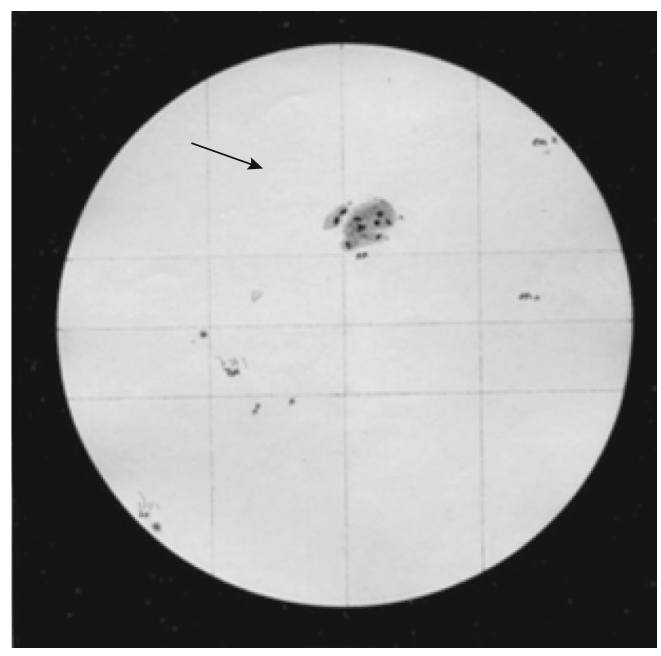

Fig. 3. View of sunspot groups Gr729 on April 29, 1882 and Gr885 on November, 1882 in SC 12 on the Greenwich Observatory filtergrams from the catalog (http://fenyi.solarobs.csfk.mta.hu/GPR/). Arrows point to the corresponding sunspot groups.

22 at 0545 UT (19 nT according to the Geomagnetic Observatory of Guam), giving rise to a magnetic storm. It increased to the values of a severe magnetic storm (G4) after the arrival of the second magnetic impulse on June 22 at 1759 UT (41 nT) from the flare on June 21 . Thus, it follows that not only complex flare events, but also the characteristics of the propagation of their disturbances in interplanetary space, can be responsible for the occurrence of extreme magnetic storms.

As follows from Table 1, the past cycle 24 is significantly inferior to SC 12 in terms of the number of magnetic storms of all classes, except for minor, G1-class magnetic storms, which are about the same (71/78). All extreme magnetic storms, except one, in SC 12 occurred during the rise branch (5/1). Such a pattern in the first epoch of lowered SA was repeated only in SC 16 (3/1). In the remaining cycles of the epoch, the ratio of extreme magnetic storms was either during the decline branch $(1 / 3)$ in SC 14 and SC 15, which is typical for the epoch of increased SA and transition periods, or equal to $2 / 2$ in SC 13 . The single severe G4 magnetic storm and all three strong G3 magnetic storms in SC 24 occurred on the declining branch. These observational facts with a high probability favor the fact that the geoeffectiveness of the propagation of a disturbance from a solar source through interplanetary space on the rise branch was significantly higher in SC 12 than in the SC 24 rise phase, while the conditions were approximately equal during the decline phase. Since there were principally more large sunspot groups ( $S p \geq 1500 \mathrm{msh}$ ) in the SC 12 rise phase (eight versus two in SC 24), and the transitional SC 11 between epochs (from increased to lowered) was high, in contrast to SC 23 (the average value), it can be assumed that the SC 12 rise branch was still under the influence of the transition period.
An example of such a possible influence of the transition period on the first cycle of the subsequent epoch is provided by the only transition period (SC 17) on a reliable SA series from the epoch of lowered SA to the epoch of increased SA, when supergiant sunspot

SDO/HMI continuum Jun. 21, 2015 0058:25 UT

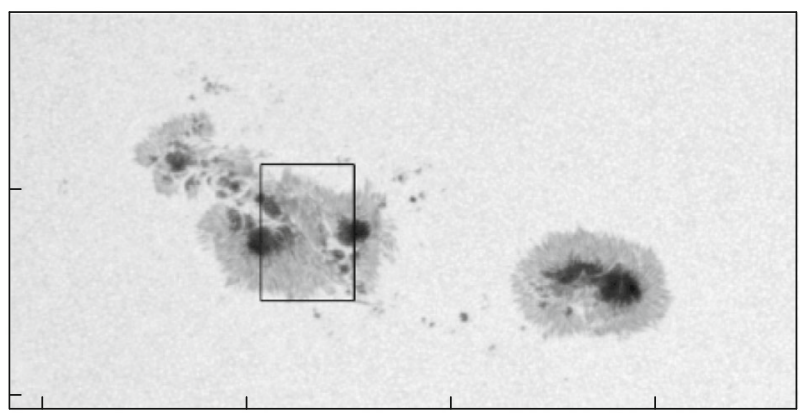

SDO/HMI longitudinal field Jun. 21, 2015 0058:25 UT

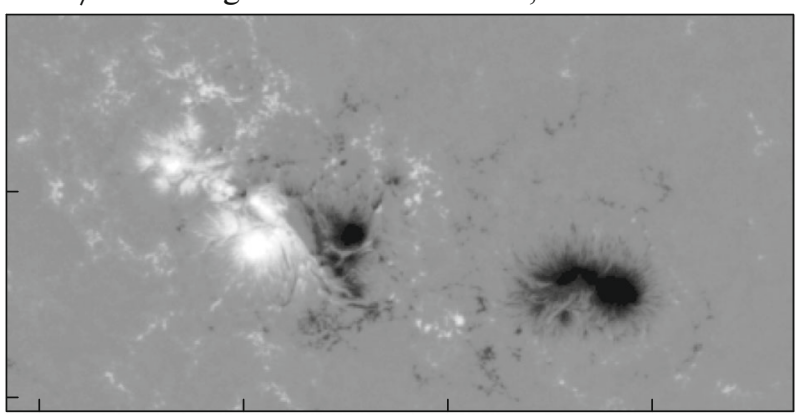

Fig. 4. View AR12371, photosphere (top panel); magnetic field (Piersanti et al., 2017) (bottom panel). The box delimits the area in which the geoeffective flare event occurred. 
groups appeared on the rise branches of SC 18 with areas $>4000 \mathrm{msh}$ for the only time in the entire history of sunspot observations; the largest area reached 6132 msh in March 1947.

\section{CONCLUSIONS}

The application of homogeneous observational data and the use of one system to estimate the intensity of disturbances in the NES for extreme (G5) and severe (G4) magnetic disturbances in the first cycles of epochs of lowered SA showed that the conditions for the implementation of highly geoeffective flare events that caused extreme magnetic storms in the NES were more favorable during the SC 12 rise branch than during the rise branch of SC 24. During the SC 24 rise branch, even strong (G3) geomagnetic disturbances were not observed. The conditions for the realization of the considered events were comparatively equal during the decline branch, but there were still significantly more of them in the first epoch of lowered SA than in the second. Only the number of minor magnetic storms (78/71) was comparatively the same, but it is known that they are mostly recurrent and that they originate from coronal holes. The asymmetry in the number of magnetic disturbances is probably a consequence of the asymmetry of sunspot-forming activity: the appearance of sunspot groups with areas $(S p \geq$ $1000 \mathrm{msh}$ ), which in our case is quite convincing: 38 appeared during the rise phase of SC 12, and only 16 occurred during the decline phase. The process is completely different in SC 24 . Only eight appeared in the rise phase of such sunspot groups, and the decline phase included six, i.e., the number of such sunspot groups was almost the same. A significant decrease in solar-activity phenomena at the beginning of the second epoch of low SA, both in sunspot-forming activity and in the occurrence of strong magnetic disturbances, may indirectly indicate higher background values of the total solar magnetic field in the first epoch (SC 12). This led to a higher level of sunspotforming activity as compared to the beginning of the second epoch of lowered SA, but, of course, a significantly lower level than that in the epoch of increased SA. This leads to the occurrence of a larger number of geoeffective flare events and, accordingly, the formation of more favorable conditions for the transmission of disturbances through the solar wind to the NES.

Thus, by the beginning of the second epoch of low SA, the changes in the solar magnetic fields and the physical conditions caused by them in the interplanetary medium turned out to be even more significant than we expected. Hence, a preliminary conclusion can be drawn that the fraction of medium-sized flare events with a complex structure, which occur in relatively small active regions with a complex magnetic configuration, was slightly higher in the initial cycle of the first epoch of lowered SA than at the present time, in the initial cycle of the second such epoch.
Therefore, along with the accumulation of observational data on the further development of the SC in the coming epoch of lowered SA, it is necessary to continue the study and to analyze carefully all of the available observational data on the SA of the first epoch, with allowance for fine variations of the geomagnetic field directly related to the solar-activity events (croche, sfe, ...), to identify features of the geoeffectiveness of active phenomena of that time and the ability to predict the development and main characteristics of SCs in the coming epoch.

\section{OPEN ACCESS}

This article is licensed under a Creative Commons Attribution 4.0 International License, which permits use, sharing, adaptation, distribution and reproduction in any medium or format, as long as you give appropriate credit to the original author(s) and the source, provide a link to the Creative Commons license, and indicate if changes were made. The images or other third party material in this article are included in the article's Creative Commons license, unless indicated otherwise in a credit line to the material. If material is not included in the article's Creative Commons license and your intended use is not permitted by statutory regulation or exceeds the permitted use, you will need to obtain permission directly from the copyright holder. To view a copy of this license, visit http://creativecommons.org/licenses/by/4.0/.

\section{REFERENCES}

Carrington, R.C., Description of a singular appearance seen in the Sun on September 1, 1859, Mon. Not. R. Astron. Soc., 1859, vol. 20, no. 1, pp. 13-15.

Coffey, H. and Erwin, E., When do the geomagnetic aa and Ap indices disagree?, J. Atmos. Sol.-Terr. Phys., 2001, vol. 63 , no. 5 , pp. $551-556$. https://doi.org/10.1016/S1364-6826(00)00171-1

Gosling, J.T., McComas, D.J., Phillips, J.L., and Bame, S.J., Geomagnetic activity associated with earth passage of interplanetary shock disturbances and coronal mass ejections, J. Geophys. Res., 1991, vol. 96, no. A5, pp. 7831-7839.

https://doi.org/10.1029/91JA00316

Ishkov, V.N., Reduced and extended periods of solar activity: Monitoring features and key facts, in Trudy konferentsii "Solnechnaya i solnechno-zemnaya fizika-2013" (Proceedings of the Conference "Solar and Solar-Terrestrial Physics-2013”), Nagovitsyn, Yu.A., Ed., St. Petersburg: WVM, 2013, pp. 111-114. http://www.gao.spb.ru/ russian/publ-s/conf_2013/conf_2013.pdf.

Ishkov, V.N., Solar flare super-events: When they can occur and the energy limits of their realization, Sun Geosphere, 2015, vol. 10, no. 1, pp. 89-96. http://newserver.stil. bas.bg/SUNGEO/00SGArhiv/SG_v10_No1_2015pp-89-96.pdf.

Ishkov, V.N., Solar sunspot-forming activity and its development on the reliable Wolf numbers series, in Variability of the Sun and Sun-like Stars: From Asteroseismology to Space Weather (EDP Sciences Proceedings), Rozelot, J.-P. and Babaev, E.S., Eds., 2018, pp. 109-118. 
Ishkov, V.N., Space weather and specific features of the development of current solar cycle, Geomagn. Aeron. (Engl. Transl.), 2018, vol. 58, no. 6, pp. 753-767.

Ishkov, V.N. and Shibaev, I.G., Solar activity cycles: General characteristics and modern forecasting boundaries, Bull. Russ. Acad. Sci.: Phys., 2006, vol. 70, no. 10, pp. 1643-1647.

Joshi, B., Ibrahim, M.S., Shanmugaraju, A., and Chakrabarty, D., A major geoeffective CME from NOAA 12371: Initiation, CME-CME interaction, and interplanetary consequences, Sol. Phys., vol. 293, id 107. https://doi.org/10.1007/s11207-018-1325-2

Livingston, W., Penn, M.J., and Svalgaard, L., Decreasing sunspot magnetic fields explain unique $10.7 \mathrm{~cm}$ radio flux, Astrophys. J. Lett., 202, vol. 757, id L8. https://doi.org/10.1088/2041-8205/757/1/L8

Mayaud, P.N., The aa indices: A 100-year series characterizing the magnetic activity, J. Geophys. Res., 1972, vol. 77, no. 34, pp. 6870-6874.

Piersanti, P., Alberti, T., and Bemporad, A., Comprehensive analysis of the geoeffective solar event of 21 June 2015: Effects on the magnetosphere, plasmasphere, and ionosphere systems, Sol. Phys., vol. 292, id 169. https://doi.org/10.1007/s11207-017-1186-0
Shibaev, I. and Ishkov, V., Investigation of the statistical characteristics of Wolf numbers reliable series: Signs of solar cycles likelihood, Proc. 7th Scientific Conf. "Space, Ecology, Safety-2011”, Sofia, Bulgaria, Nov. 29-Dec. 1, 2011, 2012, pp. 297-301. http://www.space.bas.bg/BG/magasin/SES/PROCEEDING/20SES/202011.pdf.

Srivastava, N., Shibu, K., Rohan, E., and Wiegelmann, T., Source region of the 18 November 2003 coronal mass ejection that led to the strongest magnetic storm of cycle 23, J. Geophys. Res., 2009, vol. 114, A03107. https://doi.org/10.1029/2008JA013845

Sunspot and Geomagnetic Storm Data Derived from Greenwich Observations 1874-1954, London: H. M. Stationery Office, 1955. https://doi.org/10.1002/qj.49708235223.

Svalgaard, L., The mean field of the Sun, 2011. https:// www.leif.org/research.

Vemareddy, P., Successive homologous Coronal Mass Ejections driven by shearing and converging motions in solar active region NOAA 12371, Astrophys. J., 2017, vol. 845, id 59. https://doi.org/10.3847/1538-4357/aa7ff4

Translated by E.G. Morozov 